\title{
Mask Chemical Micromachining of Cylindrical Array Structures on Copper Substrates
}

\author{
Mingqian $\mathrm{Gao}^{1, \text { a }}$, Jinlong Song ${ }^{1,2, *}$, Yao Cui $^{1, \mathrm{~b}}$, Changlin Zhao ${ }^{1, \mathrm{c}}$ \\ ${ }^{1}$ Key Laboratory for Precision and Non-Traditional Machining Technology of Ministry of Education, \\ Dalian University of Technology, Dalian 116024, China \\ ${ }^{2}$ Collaborative Innovation Center of Major Machine Manufacturing in Liaoning, Dalian University of \\ Technology, Dalian 116024, China \\ agaomingqian2013@163.com, ${ }^{\mathrm{b}} 15140443439 @ 163 . c o m,{ }^{c}$ zhaochanglin@mail.dlut.edu.cn, \\ *Corresponding author Email: songjinlong@dlut.edu.cn
}

Keywords: Microarray, Photoresist, Chemical etching, Micro-machining.

\begin{abstract}
The microarray structured surfaces in the nature, such as shark skin and lotus leaf, have good waterproof and anti-friction performances. Besides, the microarray structures above also have good application prospects in heat exchange and biomedicine. Compared to traditional machining with problems of high cost and low efficiency, the mask chemical etching method has the advantages that no residual stress exists when processing, and can be employed for large area preparation with high machining efficiency. We propose to prepare micro cylindrical array structures on copper substrate by mask chemical etching method, and research influences of processing parameters on the size of microarray structures.
\end{abstract}

\section{Introduction}

Microarray structures refer to the structures which have certain sized and neatly arranged micro protuberances or depressions on the surfaces, with superior performances on waterproof, anti-friction, heat exchange, and biomedicine[1-3]. Currently the micro array preparing methods on metal substrates still have some deficiencies. For example, wire cutting method[4] has accuracy and surface quality problems; While microarray structures can be prepared by laser etching method[5], laser devices are quite expensive; Chemical deposition method[6] can deposit heavy metals such as lead, nickel on the surfaces, but not appropriate for industrial production due to environmental concerns. According to the deficiencies of the methods above, we propose an economic and feasible method, mask chemical etching method to prepare micro cylindrical array structures on copper substrates, and determine the appropriate photolithography parameters and research on the influences of chemical etching parameters on the micro cylindrical array structure size.

\section{Experimental}

The experiment process is shown in Fig. 1. Firstly, the copper substrate $(35 \mathrm{~mm} * 35 \mathrm{~mm} * 1 \mathrm{~mm} \mathrm{~T} 2$ copper sheet, copper content more than $99.9 \%$ ) was preprocessed by washing with deionized water and acetone solution to remove oil and impurities on the substrate surface. Then mask processes are as follows. Copper substrate was coated by photoresist (BP212-37S UV positive photoresist) at a rotating speed of $450 \mathrm{rpm}$ in the beginning 15s, then the speed was increased to $2000 \mathrm{rpm}$ in the next $45 \mathrm{~s}$, for obtaining a photoresist film with uniform thickness, which was about $1.5 \mu \mathrm{m}$. Then circular array film (circle diameter is $150 \mu \mathrm{m}$ and central distance is $250 \mu \mathrm{m}$ ) as the photoresist mask was pressed tightly onto the copper substrate which was coated with the photoresist. Then, the copper substrate with mask was putted into a heating box for $40 \mathrm{~min}$ at $85^{\circ} \mathrm{C}$. The aforementioned processes need to be shielded from light. After taken out, the substrate was exposed immediately under UV light for 20s. Then the mask was removed in $0.5 \mathrm{wt} \% \mathrm{NaOH}$ solution for developing. After developing, the substrate was washed repeatedly with deionized water, for removing the residual 
developer on the surface. At last, the substrate was postbaked at $85^{\circ} \mathrm{C}$ for $1 \mathrm{~h}$ to improve the corrosion resistance of the photoresist film. Thus, the circular array photoresist mask was prepared on the copper substrate surface.

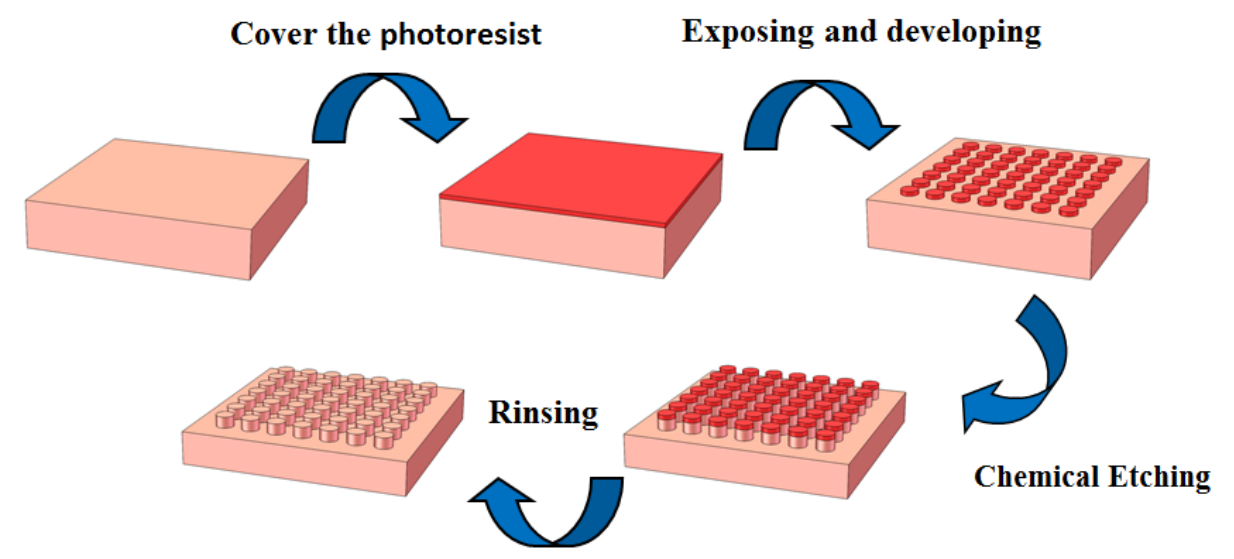

Fig. 1 The schematic of processing of mask micro machining cylindrical array structure

For obtaining micro cylindrical array structures, the copper substrate with circular array photoresist mask was etched by putting into the chemical etching solution $(2 \mathrm{~mol} / \mathrm{L} \mathrm{HCl}$ and $0.5 \mathrm{wt} \%$ $\mathrm{H}_{2} \mathrm{O}_{2}$ mixture solution), and sealed and heated at a constant temperature of $60^{\circ} \mathrm{C}$ in the heating box. The copper substrate was then taken out after etching, and rinsed with the deionized water and acetone solution, till the photoresist film on the surface was completely removed.

\section{Results and Discussion}

Developing time and etching time in the fabricating process are the vital parameters for the quality and size controlling.

After exposed for 20s, the copper substrates were putted into positive photoresist developing solution (mass fraction of $0.5 \% \mathrm{NaOH}$ solution) in the water bath at the temperature of $25^{\circ} \mathrm{C}$ for developing. The developing times were 270s, 300s and 330s, 360s and 390s respectively. After postbaking, the photoresist patterns are as shown in Fig. 2. It can be seen that when the developing time was 270s, the photoresist pattern was fuzzy, but with the developing time increasing, the photoresist pattern was presented more clearly. When the developing times were 330s, 360s and 390s, a clear photoresist circular array pattern could be obtained on the copper substrate surface.

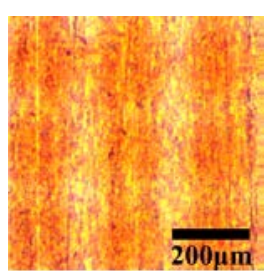

(a)270s

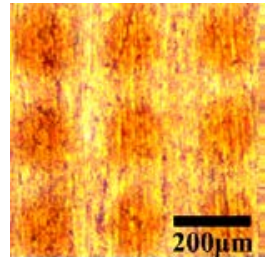

(b)300s

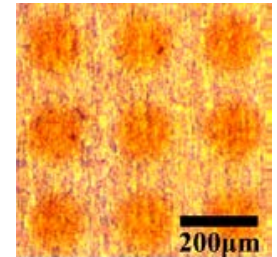

(c)330s

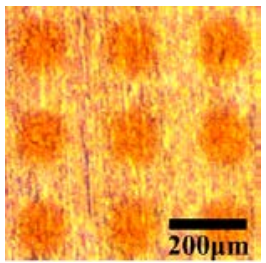

(d)360s

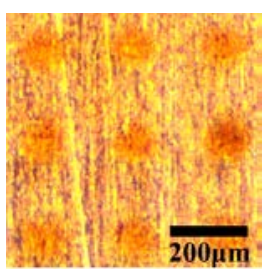

(e)390s

Fig. 2 The photoresist mask patterns under different developing time

While the clarity of the photoresist film pattern has been guaranteed, the precision of the photoresist film pattern also needs to be considered. Fig. 3 shows the influence of developing time on array circle diameter. When the exposure time was 270 s, the average array circle diameter was $207.83 \mu \mathrm{m}$, which was quite different with the designing size of $150 \mu \mathrm{m}$. The reason is that the exposed photoresist cannot be completely dissolved in the developer within insufficient developing time. With the extension of developing time, the area of exposed photoresist began dissolving in the developer gradually, and the photoresist array circle diameter became smaller and tended to the design value; when the developing time was extended to 360s, the average array circle diameter was $151.12 \mu \mathrm{m}$, very close to the design value of $150 \mu \mathrm{m}$. While the developing time was extended to 390s, the 
average array circle diameter reduced to $123.67 \mu \mathrm{m}$, far less than the design value, because the overlong developing time not only made the exposed area of photoresist completely dissolve in the solution, but also let the solution dissolve and damage the edge of the unexposed area. Therefore, it can be considered that the relatively appropriate developing time on the copper substrate is 360s, which ensures fully developing and can obtain a both clear and undamaged photoresist pattern with high dimensional accuracy.

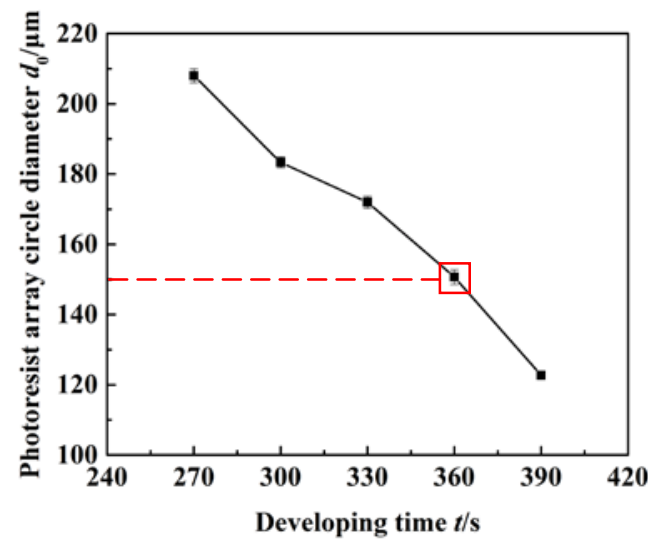

Fig. 3 The influence of developing time on array circle diameter

Then micro cylindrical array structures were prepared on copper surface by mask chemical etching method, as shown in Fig. 4. In order to research the influence of the etching time on the size of the cylindrical arrays, copper substrates covered with circular array photoresist of the above mentioned design size were putted into $2 \mathrm{~mol} / \mathrm{L} \mathrm{HCl}$ and mass fraction of $0.5 \% \mathrm{H}_{2} \mathrm{O}_{2}$ mixture chemical etching solution, under the temperature of $60^{\circ} \mathrm{C}$, for $1 \mathrm{~h}, 2 \mathrm{~h}, 3 \mathrm{~h}, 4 \mathrm{~h}, 5 \mathrm{~h}$ and $6 \mathrm{~h}$ respectively.

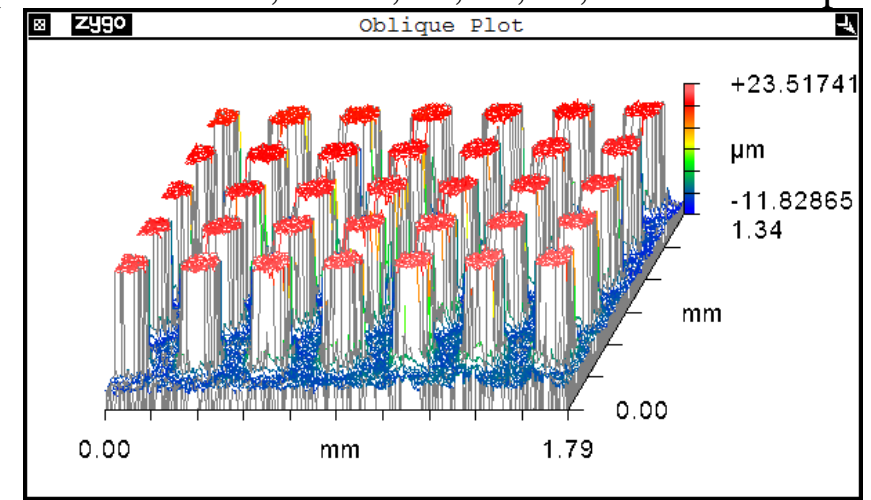

Fig. 4 The micro cylindrical array structures on copper surface by mask chemical etching method (Etching time 1h)

Etching time has a great influence on the structure. As shown in Fig. 5, it can be seen that the average diameter of cylindrical arrays decreased from $134.24 \mu \mathrm{m}$ at $1 \mathrm{~h}$ to $37.36 \mu \mathrm{m}$ at $5 \mathrm{~h}$, while the average height increased from $18.58 \mu \mathrm{m}$ at $1 \mathrm{~h}$ to $60.66 \mu \mathrm{m}$ at $5 \mathrm{~h}$, and then decreased to $43.72 \mu \mathrm{m}$ at $6 \mathrm{~h}$ with the etching process going on. With the chemical reaction continued, the corrosion of copper increased, so the average diameter of cylindrical arrays kept decreasing and the average height kept increasing. While the removal volume came to the point where the diameter of cylindrical arrays was too small to hold the photoresist film above, the film fell out, and the top of the cylindrical structure began to react, making the cylindrical array structures become smaller and shorter. 

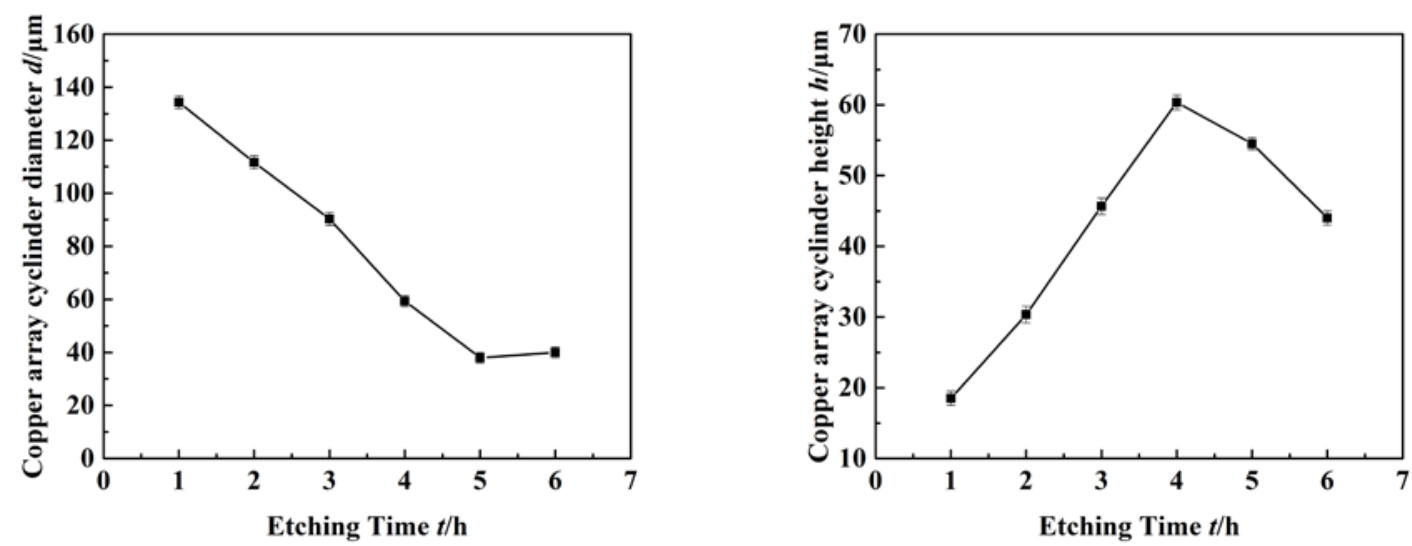

Fig. 5 The influence of etching time on array of cylinder diameter and height

\section{Conclusions}

We prepared micro cylindrical array structures on copper surface by mask chemical etching method, and discussed the influences of photolithography parameters on the photoresist pattern and chemical etching parameters on cylindrical array microstructure size. A clear and complete photoresist pattern on the copper substrate can be prepared in the appropriate developing time of about 360s. The mask pattern size will be larger than the design value if the developing time is shorter than 360 s, while the mask pattern size will be smaller and incomplete if the developing time is too long since the design edge is damaged. In the process of chemical etching, we also found that with the etching time extending, the removal increases, accompanied with a decreasing cylindrical array diameter and an increasing cylindrical array height. However, if the etching time is too long, when the cylindrical array diameter is down to about $40 \mu \mathrm{m}$, the top of the cylinder structure will be damaged, resulting in shortening of the cylindrical array height. Thus, since learning the chemical etching laws of copper, we can control the size of the micro cylindrical array conveniently and effectively by changing the etching time, which can be applied into various environments.

\section{Acknowledgments}

This project was financially supported by National Natural Science Foundation of China (NSFC, Grant No.51605078) and Science Fund for Creative Research Groups of NSFC (51621064).

\section{References}

[1] Han, X. and D. Zhang, Study on the micro-replication of shark skin. Science in China Series E: Technological Sciences, 2008. 51(7): p. 890-896.

[2] Silk, E.A., J. Kim and K. Kiger, Spray cooling of enhanced surfaces: Impact of structured surface geometry and spray axis inclination. International Journal of Heat and Mass Transfer, 2006. 49(25-26): p. 4910-4920.

[3] Lee, S., et al., Influence of microgroove dimension on cell behavior of human gingival fibroblasts cultured on titanium substrata. Clinical Oral Implants Research, 2009. 20(1): p. 56-66.

[4] Bae, W.G., et al., One-Step Process for Superhydrophobic Metallic Surfaces by Wire Electrical Discharge Machining. ACS APPLIED MATERIALS \& INTERFACES, 2012. 4(7): p. 3685-3691.

[5] Etsion, I., Improving tribological performance of mechanical components by laser surface texturing. TRIBOLOGY LETTERS, 2004. 17(4): p. 733-737.

[6] Sellmyer, D.J., M. Zheng and R. Skomski, Magnetism of Fe, Co and Ni nanowires in self-assembled arrays. 2001. 13: p. R433. 